\title{
A Single Base Substitution in the Coding Region for Neurophysin II Associated with Familial Central Diabetes Insipidus
}

\author{
Masafumi Ito, Yuichi Mori, Yutaka Oiso, and Hidehiko Saito \\ The First Department of Internal Medicine, Nagoya University School of Medicine, Nagoya 466, Japan
}

\begin{abstract}
To elucidate the molecular mechanism of familial central diabetes insipidus (FDI), we sequenced the arginine vasopressinneurophysin II (AVP-NPII) gene in 2 patients belonging to a pedigree that is consistent with an autosomal dominant mode of inheritance. 10 patients with idiopathic central diabetes insipidus (IDI) and 5 normals were also studied. The AVP-NPII gene, locating on chromosome 20 , consists of three exons that encode putative signal peptide, AVP, NPII, and glycoprotein. Using polymerase chain reaction, fragments including the promoter region and all coding regions were amplified from genomic DNA and subjected to direct sequencing. Sequences of 10 patients with IDI were identical with those of normals, while in 2 patients with FDI, a single base substitution was detected in one of two alleles of the AVP-NPII gene, indicating they were heterozygotes for this mutation. It was a $G \rightarrow A$ transition at nucleotide position 1859 in the second exon, resulting in a substitution of Gly for Ser at amino acid position 57 in the NPII moiety. It was speculated that the mutated AVP-NPII precursor or the mutated NPII molecule, through their conformational changes, might be responsible for AVP deficiency. (J. Clin. Invest. 1991. 87:725-728.) Key words: arginine vasopressin-neurophysin II gene - polymerase chain reaction • direct sequencing $\bullet$ arginine vasopressin $\bullet$ idiopathic central diabetes insipidus
\end{abstract}

\section{Introduction}

Central diabetes insipidus (CDI) ${ }^{1}$ is a disorder of impaired water conservation which results from a deficiency of arginine vasopressin (AVP). According to the causes, CDI can be classified into three categories, familial, idiopathic, and secondary. Familial CDI (FDI) is an extremely rare disorder, usually transmitted as an autosomal dominant trait $(1,2)$, while idiopathic CDI (IDI) is far more common, comprising $30 \%$ of CDI (3).

The arginine vasopressin-neurophysin II (AVP-NPII) gene assigned to human chromosome 20 (4) consists of three exons that are separated by two introns (5). This structural organization of the gene is conserved among most vertebrate species $(6$,

Address correspondence and reprint requests to Dr. Masafumi Ito, The First Department of Internal Medicine, Nagoya University School of Medicine, 65 Tsurumai-cho, Showa-ku, Nagoya, 466 Japan.

Received for publication 23 August 1990 and in revised form 11 October 1990.

1. Abbreviations used in this paper: AVP-NPII, arginine vasopressinneurophysin II; CDI, central diabetes insipidus; FDI, familial central diabetes insipidus; IDI, idiopathic central diabetes insipidus.

J. Clin. Invest.

(C) The American Society for Clinical Investigation, Inc.

$0021-9738 / 91 / 02 / 0725 / 04 \$ 2.00$

Volume 87, February 1991, 725-728
7). The first exon (exon 1) encodes putative signal peptide, AVP, and the $\mathrm{NH}_{2}$-terminal region of neurophysin II (NPII); the second exon (exon 2) accounts for the central region of NPII; and the third exon (exon 3) gives rise to the $\mathrm{COOH}$-terminal region of NPII and glycoprotein. The three exons are transcribed to mRNA, which is then translated to form an AVP-NPII precursor. This precursor undergoes posttranslational processing during its transport to axonal terminals within neurosecretory vesicles to yield AVP, NPII, and glycoprotein (8).

The homozygous Brattleboro rat, characterized by an autosomal recessive transmission of CDI (9) has been shown to have a single base deletion in exon 2 of the AVP-NPII gene (10). The nucleotide deletion causes frame shift and extinguishes a stop codon and a glycosylation site, resulting in an AVP-NPII precursor with an altered $\mathrm{COOH}$-terminal region. Translation of the poly(A)-tail yields a highly charged polylysine sequence, which is suggested to impair the transport or processing of the precursor $(11,12)$.

With respect to FDI, it has recently been suggested that there exists a genetic locus responsible for the disease within or near the AVP-NPII gene from the results of linkage study performed on two pedigrees (13).

In this study, we sequenced the AVP-NPII gene in two patients with FDI. Patients with IDI and normal subjects were also included in this study. Direct sequencing was performed on the PCR-amplified DNA, and the sequence ranging from the promoter region to the 3 '-untranslated region except for two introns was determined. Significance of a mutation found in patients with FDI is discussed.

\section{Methods}

Subjects. 2 patients belonging to a pedigree of FDI and 10 patients with IDI were studied. Patients were diagnosed as CDI based on the clinical features of excessive polyuria, polydipsia, and thirst, reduced levels of plasma AVP, and marked responses to exogenous vasopressin. AVP responses to hypertonic saline infusion were impaired in these patients. In the pedigree of FDI, 6 members spanning 3 generations were supposed to be affected, judging from symptoms such as polyuria and polydipsia (Fig. 1). Accordingly, this pedigree was consistent with an autosomal dominant mode of inheritance which is usually observed in the reported pedigrees of FDI $(1,2)$. Basal plasma AVP levels of the 2 patients studied were 0.26 and $0.36 \mathrm{pg} / \mathrm{ml}$ (III-1 and III-2, respectively). The 2 patients and their 2 affected siblings had noticed symptoms in their childhood or teens. Although it must be a significant value to study unaffected subjects in this pedigree, specimens were not available. 10 patients with IDI were subjected to computed tomography and/or magnetic resonance imaging to preclude secondary diabetes insipidus. 5 of them were diagnosed when they were under $10 \mathrm{yr}$ of age. To ascertain the nucleotide sequence of the AVP-NPII gene previously reported (5), normal subjects of 3 Japanese and 2 Caucasians were also included in the sequencing analysis. Another 20 unrelated normal Japanese were subjected to the restriction enzyme analysis described below. Informed consent was obtained from patients and normal individuals.

Primers. On the basis of the genomic sequence of the AVP-NPII gene (5), nine oligonucleotides shown in Table I were synthesized using 
Figure 1. Pedigree of a family with FDI.

Affected members are shown by filled symbols.

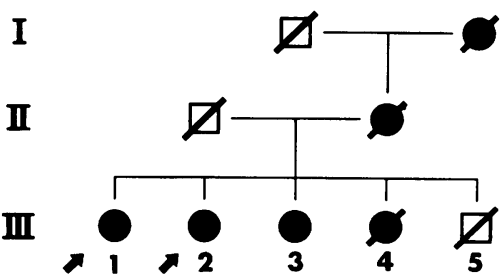

Two patients studied

(III-1, III-2) are

indicated by arrows.

Oblique lines represent

deceased members.

Squares and circles

indicate males and

females, respectively.

a DNA synthesizer (model 391A; Applied Biosystems, Inc., Foster City, CA) and were used as PCR and/or sequencing primers. Nucleotide was numbered according to the previous report (5).

Amplification of genomic DNA using PCR. Genomic DNA was extracted from whole blood as described previously (14). Using the method of Saiki et al. (15) with minor modifications, PCR was performed in a $50-\mu l$ reaction volume containing $1 \mu \mathrm{g}$ of purified genomic DNA, $1.5 \mathrm{mM}$ of each deoxynucleotide triphosphate, $67 \mathrm{mM}$ Tris$\mathrm{HCl}, \mathrm{pH} 8.8,6.7 \mathrm{mM} \mathrm{MgCl}_{2}, 16.6 \mathrm{mM}$ ammonium sulfate, $10 \mathrm{mM}$ 2-mercaptoethanol, 10\% DMSO, 100 pmol of each PCR primer, and $2.5 \mathrm{U}$ of Taq DNA polymerase (Perkin-Elmer Cetus Corp., Norwalk, CT). The reaction was carried out using Perkin-Elmer Cetus Thermal Cycler (model PJ 1000) with a temperature cycle of 1 min at $94^{\circ} \mathrm{C}, 1$ min at $60^{\circ} \mathrm{C}$, and $3 \mathrm{~min}$ at $72^{\circ} \mathrm{C}$. After 40 cycles, the amplified materials were applied to $3 \%$ NuSieve agarose gel electrophoresis (FMC BioProducts, Rockland, ME) and desired fragments were separated for sequencing.

Direct sequencing of double-stranded DNA. Double-stranded DNA template ( $0.8 \mathrm{pmol})$ was alkali-denatured as previously described (16). Subsequent reactions were performed mainly according to the manufacturer's instructions (Sequenase kit; United States Biochemical Corp., Cleveland, OH), using $\alpha-{ }^{35}$ S-dCTP (Amersham Corp., Arlington Heights, IL) and 7-deaza dGTP (Boehringer Mannheim Inc., Indianapolis, IN). The denatured template was annealed with $4 \mathrm{pmol}$ of sequencing primer, followed by labeling and termination reactions. The samples were analyzed by electrophoresis on $6 \%$ polyacrylamide $/ 8$ $M$ urea gel. To obtain accurate sequencing results, both strands were sequenced using multiple different primers (Table I, Fig. 2). Major modifications from manufacturer's instructions were inclusion of $10 \%$ DMSO throughout annealing, labeling, and termination reactions (17), and a moderately lower temperature at the labeling reaction $\left(16^{\circ} \mathrm{C}\right)$.

Digestion of amplified DNA by restriction enzyme. PCR-amplified fragment isolated from $3 \%$ NuSieve agarose was digested with Msp I (Boehringer Mannheim Inc., Indianapolis, IN) according to the manufacturer's instruction and analyzed on $8 \%$ polyacrylamide gel. The gel was stained with ethidium bromide before visualization under ultraviolet light.

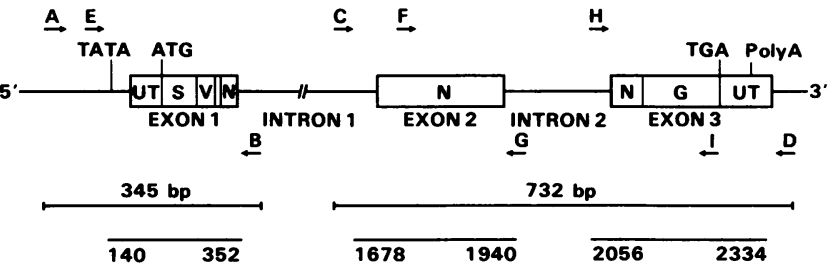

Figure 2. Schematic representation of the AVP-NPII gene and the strategy for PCR and direct sequencing. Exon 1, 2, and 3 (open boxes), and locations of TATA box (TATA), translation initiation $(A T G)$ and termination $(T G A)$ signals, polyadenylation signal (Poly $A$ ), and primers (listed in Table $\mathrm{I})$ are indicated. Coding regions for the signal sequence $(S), \operatorname{AVP}(V)$, NPII $(N)$, and glycoprotein $(G)$ are shown. PCR-amplified fragments of 345 and 732 bp used for sequencing are indicated in the middle of the figure. Solid lines at the bottom show the range sequenced in this study.

\section{Results}

In all 17 subjects, PCR-amplification using primer $A$ and $B$, and $C$ and $D$ resulted in DNA fragments of expected sizes, 345 and 732 bp, respectively (Table I, Fig. 2) (data not shown). The former included the promoter region, exon 1 and its adjacent intron 1, the latter exon 2 , its adjacent intron 1 , intron 2 , and exon 3. Therefore, no major insertions and deletions were implicated in the regions of the AVP-NPII gene described above.

Double-stranded DNAs of 345 and 732 bp were directly sequenced by use of primer $A, B$, and $E$, and primer $C, D, F, G$, $H$, and $I$, respectively (Table I, Fig. 2). Of the nine primers, five (primer $E, F, G, H$, and $I$ ) were used as internal nested primers. As shown in Fig. 2, we sequenced the AVP-NPII gene spanning bases 140 to 352 (the promoter region, exon 1, and its adjacent intron 1), 1678 to 1940 (exon 2 and its adjacent introns 1 and 2) and 2056 to 2334 (exon 3 and its adjacent intron 2).

Nucleotide sequences of the signal sequence and the 3 '-untranslated region were identical in all 17 subjects, in which two normal Caucasians were included, but showed several discordances with those previously described (5) (Fig. 3). Then, the amino acid residue Pro $(C C T)$ in the signal peptide was corrected to Leu (CTC).

Sequences of 10 patients with IDI showed no difference from those of normals, while bands corresponding to both $G$ and $A$ were visualized at nucleotide position 1859 in 2 patients (III-1, III-2) with FDI (Fig. 4). This mutation was a $\mathrm{G} \rightarrow \mathrm{A}$ transition in exon 2 and the patients were supposed to be heterozygotes.

Table I. Sequences and Locations of Oligonucleotide Primers

\begin{tabular}{|c|c|c|}
\hline Primer & Sequence & Location \\
\hline A & 5'-TGCCTGAATCACTGCTGACCGCTGGGGACC-3' & (nt38 $\rightarrow$ 67; 5'flanking) \\
\hline B & 5'-GCTATGGCTGCCCTGAGATGGCCCACAGTG-3' & $($ nt382 $\rightarrow 353 ;$ intron 1$)$ \\
\hline C & 5'-TCGCTGCGTTCCCCTCCAACCCCTCGACTC-3' & (nt1648 $\rightarrow 1677 ;$ intron 1$)$ \\
\hline D & 5'-ССТСТСТССССТTСССТCTTCCCGCCAGAG-3' & (nt2379 $\rightarrow 2350 ; 3$ 'flanking) \\
\hline $\mathbf{E}$ & 5'-TGGCGGCCGCGTCTCGCCTCCACGGGAACA-3' & (nt107 $\rightarrow$ 136; 5'flanking) \\
\hline $\mathbf{F}$ & 5'-TGCTTCGGGCCCAGCATCTGCTGCG-3' & $($ nt1751 $\rightarrow 1775 ;$ exon 2$)$ \\
\hline $\mathbf{G}$ & 5'-CGCCCCCCCCCAGGCCCGCCCCCGCCGCGC-3' & (nt1951 $\rightarrow$ 1922; intron 2 ) \\
\hline $\mathrm{H}$ & 5'-CCCAGGCGCCCGTGCTCACACGTCCTCCCG-3' & (nt2053 $\rightarrow$ 2082; intron 2 ) \\
\hline I & 5'-TAGGCGTCGGGCTGGGCGGGCTCGA-3' & $(\mathrm{nt} 2255 \rightarrow 2231 ;$ exon 3 ) \\
\hline
\end{tabular}

Nucleotide $(n t)$ is numbered according to the previously reported sequence (5). Primers A, C, E, F, and H correspond to the sequences of the sense strand; primers B, D, G, and I correspond to those of the antisense strand. 
Signal sequence

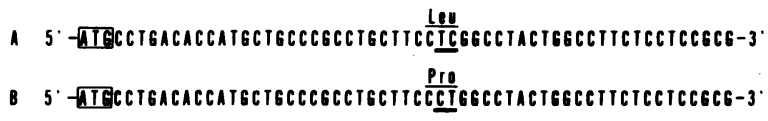

\section{3'- untranslated region}

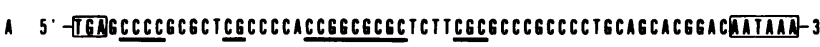

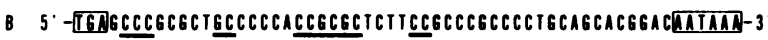

Figure 3. Corrected sequences of the signal sequence and the 3'untranslated region. Differences between sequences determined in this study $(A)$ and those of a previous report $(5)(B)$ are underlined. Translation initiation $(A T G)$ and termination $(T G A)$ signals, and polyadenylation signal (AATAAA) are indicated (open boxes). The amino acid residue Pro $(C C T)$ in the signal peptide is corrected to Leu $(C T C)$.

According to the mutation, a restriction site of Msp I was expected to disappear ( $C C G G \rightarrow C C A G$ ) (Fig. 4). To rule out sequencing artifacts, the PCR-amplified fragment of $304 \mathrm{bp}$, including the mutation site, was subjected to the restriction enzyme analysis. As illustrated in Fig. $5 A$, it was anticipated that the digest of PCR product from the normal allele should be four fragments $(123,93,56$, and $32 \mathrm{bp})$, whereas that from the mutant allele, three $(216,56$, and $32 \mathrm{bp})$. Namely, the abolishment of Msp I site in the mutant allele was expected to yield a 216-bp fragment, instead of 123- and 93-bp fragments generated from the normal allele. On the electrophoretic analysis, bands of 216,123 , and $93 \mathrm{bp}$ were detected in the patients (III-1, III-2), indicating that they had both of the mutant and normal alleles (Fig. $5 B$ ). Accordingly, it was confirmed that the patients were heterozygotes for this mutation. The heterozygosity was compatible with an autosomal dominant hereditary pattern in the pedigree.

The same restriction enzyme analysis was also performed on 20 unrelated normal Japanese, and none of them had the 216-bp fragment specific for the mutant allele (data not shown). In addition, 10 patients with IDI and 5 normals sequenced in this study did not carry the mutation found in patients with FDI. Thus, we examined 70 alleles other than those of patients with FDI and no allele was found to have the mutation.
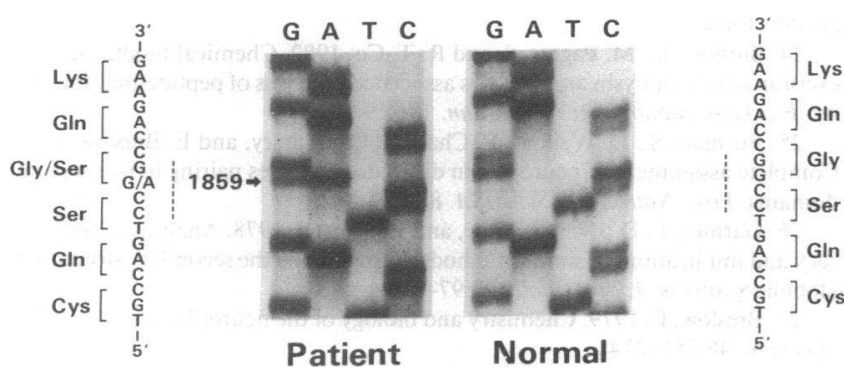

Figure 4. Direct sequencing of PCR-amplified DNA. In a patient (III-1), a band corresponding to $A$ other than $G$ is observed at nucleotide position 1859 (arrow), indicating the patient is a heterozygote for the mutation. This mutation is expected to alter Gly $(G G C)$ to $\operatorname{Ser}(A G C)$ at amino acid position 57 in the NPII moiety. The tetranucleotide sequence (CCGG) recognized by Msp I is shown (broken line).
A

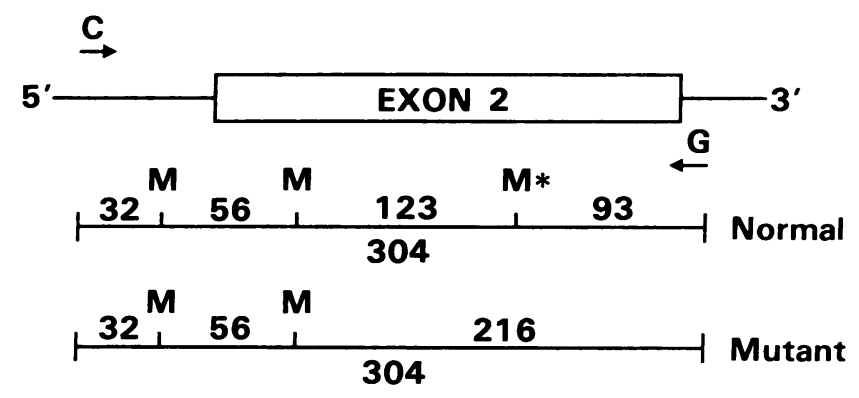

B

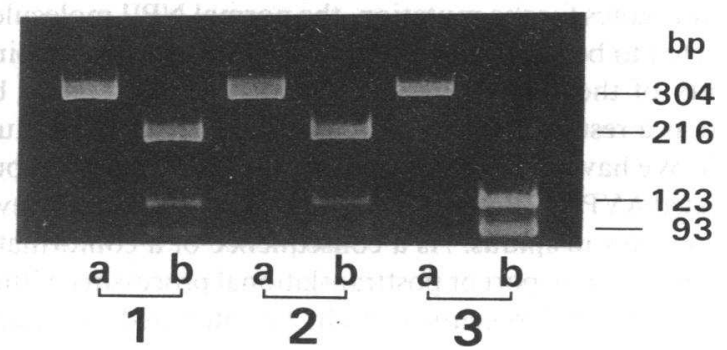

Figure 5. Restriction enzyme digestion of PCR-amplified DNA. $(A)$ Restriction sites of Msp I in PCR-amplified 304-bp fragment including the mutation site are indicated $(M)$. One of those found in the normal allele is abolished in the mutant allele according to the nucleotide substitution $(C C G G \rightarrow C C A G)(*)$. As a result, the mutant allele is expected to yield a 216-bp fragment, instead of 123- and 93-bp fragments generated from the normal allele. $(B)$ Undigested $(a)$ and digested $(b)$ materials are applied to $8 \%$ polyacrylamide gel. A 216-bp fragment specific for the mutant allele is detected in two patients (III- 1 and III-2, lanes $l$ and 2, respectively) with FDI in addition to 123- and 93-bp fragments found in a normal subject (lane 3 ), indicating the patients are heterozygotes for the mutation.

\section{Discussion}

A single base substitution was detected on one of two alleles of the AVP-NPII gene in two patients in a pedigree with FDI. Therefore, they were judged as heterozygotes for this mutation. It was $\mathrm{a} G \rightarrow \mathrm{A}$ transition at nucleotide position 1859 in exon 2 , predicting a substitution of Gly $(G G C)$ for polar $\operatorname{Ser}(A G C)$ at amino acid position 57 in the NPII moiety. This mutation was expected to abolish Msp I site in the AVP-NPII gene. Although a segregation of the mutation in the pedigree could not be studied, the possibility of the mutation due to polymorphism was very small. Therefore, it was speculated that the mutation might be responsible for AVP deficiency in these patients with FDI. To our knowledge, this is the first report demonstrating a mutation in the AVP-NPII gene as a possible cause for human FDI. It will be interesting to examine whether the same mutation is also detected in other pedigrees with FDI. Although the restriction enzyme analysis of the AVP-NPII gene was recently reported in Caucasian pedigrees with FDI, unfortunately, Msp I was not included in the study (13).

The NPII molecule consists of internally duplicated regions $\left(\mathrm{NH}_{2}\right.$ domain, residues $12-31$; $\mathrm{COOH}$ domain, $\left.60-77\right)$ that are separated by the evolutionally conserved nonduplicated region (18). NPII binds AVP and self associates to form the dimer complex and the dimerization of NPIIs leads to their increased 
binding affinity with AVP (19-21). Although regions of NPII participating in self association are still unknown, Tyr-49 and Arg-8 in NPII are reported to be involved in AVP-binding (2224). Also, the disulfide bond, Cys-10 to Cys-54, encompassing the $\mathrm{NH}_{2}$ domain is supposed to be critical for AVP-binding (25). As Gly-57 is a conserved amino acid among most vertebrate species (18), its substitution could influence AVP-binding and/or self association indirectly through a conformational change of the NPII molecule. Indeed, computer-assisted prediction of the secondary structure by the method of Robson et al. (26) indicated the possibility of a conformational change in the NPII molecule due to the substitution of Gly-57 for Ser. Consequently, supposed functions of NPII such as protecting AVP from proteolytic degradation and assisting AVP in its axonal transport (27) might be impaired. As the patients are heterozygotes for the mutation, the normal NPII molecule also expected to be generated in their hypothalamus. AVP-binding affinity of the normal NPII molecule, however, could be altered as a result of a heterodimer formation with the mutated NPII. We have focused on the mutated NPII molecule, but the mutated AVP-NPII precursor might also participate in developing diabetes insipidus. As a consequence of a conformational change, the transport or posttranslational processing of the mutated AVP-NPII precursor might be interfered. To elucidate precise mechanisms how the mutation found in the NPII moiety is responsible for AVP deficiency, further studies including in vitro expression of constructs having the substitution in cultured cells will be required, which will also contribute to our understanding of NPII.

In this study, we have encountered some difficulties in PCR-amplification and direct sequencing, because the AVPNPII gene is extremely $G+C$ rich (5). It manifests in the latter half of the gene including exon 2 , intron 2 , and exon 3 which are composed of 74,84 , and $75 \%$ of $G+C s$, respectively. We have overcome the difficulties by inclusion of DMSO during PCR and sequencing reaction, whose effect is presumably due to the prevention of the secondary structure formation. The reason why the previously reported sequence of the 3'-untranslated region differed from that determined in this study is supposed to be $83 \%$ of $\mathrm{G}+\mathrm{Cs}$ in this region.

The etiology of IDI is still unknown, although autoimmunity was supposed to be a candidate in some cases (28). As IDI might possibly be caused by some genetic defects, sequencing analysis of the AVP-NPII gene was performed on 10 patients of which 5 were juvenile onset. However, no difference was found compared with normal subjects. To elucidate the molecular basis of IDI, flanking regions of the AVP-NPII gene and factors influencing its expression must be analyzed.

\section{References}

1. Baylis, P. H., and G. L. Robertson. 1981. Vasopressin function in familia cranial diabetes insipidus. Postgrad. Med. J. 57:36-40.

2. Kaplowitz, P. B., A. J. D'Ercole, and G. L. Robertson. 1982. Radioimmunoassay of vasopressin in familial central diabetes insipidus. J. Pediatr. 100:76-81.

3. Moses, A. M., and D. D. Notman. 1982. Diabetes insipidus and syndrome of inappropriate antidiuretic hormone secretion (SIADH). Adv. Intern. Med. 27:73-100.

4. Riddell, D. C., R. Mallonee, J. A. Phillips, J. S. Parks, L. A. Sexton, and J. L.
Hamerton. 1985. Chromosomal assignment of human sequences encoding arginine vasopressin-neurophysin II and growth hormone releasing factor. Somat. Cell. Mol. Genet. 11:189-195.

5. Sausville, E., D. Carney, and J. Battey. 1985. The human vasopressin gene is linked to the oxytocin gene and is selectively expressed in a cultured lung cancer cell line. J. Biol. Chem. 260:10236-10241.

6. Schmale, H., S. Heinsohn, and D. Richter. 1983. Structural organization of the rat gene for the arginine vasopressin-neurophysin precursor. EMBO (Eur. Mol. Biol. Organ.) J. 2:763-767.

7. Land, H., G. Schütz, H. Schmale, and D. Richter. 1982. Nucleotide sequence of cloned cDNA encoding bovine arginine vasopressin-neurophysin II precursor. Nature (Lond.). 295:299-303.

8. Brownstein, M. J., J. T. Russel, and H. Gainer. 1980. Synthesis, transport, and release of posterior pituitary hormones. Science (Wash. DC). 207:373-378. 9. Sokol, H. W., and H. Valtin. 1982. The Brattleboro rat. Ann. NY. Acad. Sci. 394:1-828.

10. Schmale, H., and D. Richter, 1984. Single base deletion in the vasopressin gene is the cause of diabetes insipidus in Brattleboro rats. Nature (Lond.). 308:705-709.

11. Krisch, B., P. Nahke, and D. Richter, 1986. Immunocytochemical staining of supraoptic neurons from homozygous Brattleboro rats by use of antibodies against two domains of the mutated vasopressin precursor. Cell Tissue. Res. 244:351-358

12. Guldenaar, S. E. F., P. Nahke, and B. T. Pickering. 1986. Immunocytochemical evidence for the presence of a mutant vasopressin precursor in the supraoptic nucleus of the homozygous Brattleboro rat. Cell Tissue. Res. 244:431 436.

13. Repaske, D. R., J. A. Phillips III, L. T. Kirby, W. J. Tze, A. J. D'Ercole, and J. Battey. 1990. Molecular analysis of autosomal dominant neurohypophyseal diabetes insipidus. J. Clin. Endocrinol. Metab. 70:752-757.

14. Ito, M., M. Tanimoto, H. Kamura, M. Yoneda, Y. Morishima, K. Yamauchi, T. Itatsu, K. Takatsuki, and H. Saito. 1989. Association of HLA antigen and restriction fragment length polymorphism of $T$ cell receptor $\beta$-chain gene with Graves' disease and Hashimoto's thyroiditis. J. Clin. Endocrinol. Metab. 69:100-104.

15. Saiki, R. K., D. H. Gelfand, S. Stoffel, S. J. Scharf, R. Higuchi, G. T. Horn, K. B. Mullis, and H. A. Erlich. 1988. Primer-directed enzymatic amplification of DNA with a thermostable DNA polymerase. Science (Wash. DC). 239:487-491.

16. Haltiner, M. T. Kempe, and R. Tjian. 1985. A novel strategy for constructing clustered point mutations. Nucleic Acids Res. 13:1015-1025.

17. Winship, P. R. 1989. An improved method for directly sequencing PCR amplified material using dimethyl sulphoxide. Nucleic Acids Res. 17:1266.

18. North, W. G. 1987. Biosynthesis of vasopressin and neurophysins. In Vasopressin. Principles and properties. D. M. Gash, and G. J. Boer, editors. Plenum Publishing Corp., New York. 175-209.

19. Rholam, M., P. Nicolas, and P. Cohen. 1982. Binding of neurohypophyseal peptides to neurophysin dimer promotes formation of compact and spherical complexes. Biochemistry. 21:4968-4973.

20. Fassina, G., and I. M. Chaiken. 1988. Structural requirements of peptide hormone binding for peptide-potentiated self-association of bovine neurophysin II. J. Biol. Chem. 263:13539-13543.

21. Balaram, P. A. A. Bothner-By, and E. Breslow. 1973. Nuclear magnetic resonance studies of the interaction of peptides and hormones with bovine neurophysin. Biochemistry. 12:4695-4704.

22. Peyton, D., V. Sardana, and E. Breslow. 1986. Dimerization of native and proteolytically modified neurophysins as monitored by proton magnetic resonance spectroscopy: proximity of tyrosine- 49 to the subunit interface. Biochemis try. 25:6579-6586.

23. Sardana, V., J. D. Carlson, E. Breslow, and D. Peyton. 1987. Chemical modification and cross-linking of neurophysin tyrosine-49. Biochemistry. 26:995-1003.

24. Breslow, E., M. Pagnozzi, and R. T. Co. 1982. Chemical modification or excision of neurophysin arginine-8 is associated with loss of peptide-binding ability. Biochem. Biophys. Res. Commun. 106:194-201.

25. Burman, S., D. Wellner, B. Chait, T. Chaudhary, and E. Breslow. 1989. Complete assignment of neurophysin disulfides indicates pairing in two separate domains. Proc. Natl. Acad. Sci. USA. 86:429-433.

26. Garnier, J., D. J. Osguthorpe, and B. Robson. 1978. Analysis of the accuracy and implications of simple methods for predicting the secondary structure of globular proteins. J. Mol. Biol. 120:97-120.

27. Breslow, E. 1979. Chemistry and biology of the neurophysins. Annu. Rev. Biochem. 48:251-274.

28. Scherbaum, W. A., and G. F. Bottazzo. 1983. Autoantibodies to vasopressin cells in idiopathic diabetes insipidus: evidence for an autoimmune variant. Lancet. i:897-901. 